\title{
A General Class of Quantum Fields without Cut-offs in Two Space-Time Dimensions*
}

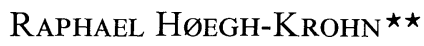 \\ Joseph Henry Laboratories, Princeton University, Princeton, N.J.
}

Received May 27, 1970/February 15, 1971

\begin{abstract}
We consider a selfinteracting boson field in two space-time dimensions, with interaction densities of the form: $V(\varphi(x))$ : where $\varphi(x)$ is a scalar boson field, and $V(\alpha)$ is a real positive function of exponential type. We define the space cut-off interaction by $V_{r}=\int_{|x| \leqq r}: V(\varphi(x)): d x$ and prove that $H_{r}=H_{0}+V_{r}$, where $H_{0}$ is the free energy, is essentially self adjoint. This permits us to take away the space cut-off and we obtain a quantum field free of cut-offs.
\end{abstract}

\section{Introduction}

Let $H_{0}$ be the free energy of a scalar boson field $\varphi(x)$ of strictly positive mass $m$ in one space dimension, so that $x \in R$. We consider the operator $H=H_{0}+V$, where $V$ is a space cut-off local interaction formally given by

$$
V=\int_{|x| \leqq r}: V(\varphi(x)): d x .
$$

Here $V(\alpha)$ is a positive real function of the form

$$
V(\alpha)=\int e^{\alpha s} d v(s),
$$

where $v$ is a positive measure on the real line with support in an interval of form $\{s:|s|<\sqrt{2 \pi}-\varepsilon\}$ with $\varepsilon>0$. : $V(\varphi(x))$ : stands for the Wick ordered function of $\varphi(x)$, and we shall return to the definition of this later. $H_{0}$ is a self adjoint operator on the boson Fock space $\mathscr{F}$ which is a direct sum $\mathscr{F}=\sum_{n=0}^{\infty} \mathscr{F}^{n}$ where $\mathscr{F}^{n}$ is the space of symetric square integrable functions of $n$ (momentum) variables. The field $\varphi(x)$ is given in terms of the annihilation-creation operators $a^{*}$ and $a$ on $\mathscr{F}$ by

$$
\varphi(x)=(4 \pi)^{-\frac{1}{2}} \int e^{i k x}\left[a^{*}(-k)+a(k)\right] \mu(k)^{-\frac{1}{2}} d k
$$

* At leave from Mathematical Institute, Oslo University.

$\star \star$ This research partially sponsored by the Air Force Office of Scientific Research under Contract AF 49(638)1545. 
where $\mu(k)=\left(k^{2}+m^{2}\right)^{\frac{1}{2}}$. The annihilation-creation operators satisfy

$$
\left[a(k), a^{*}\left(k^{\prime}\right)\right]=\delta\left(k-k^{\prime}\right) .
$$

We shall also use the notation $\varphi_{+}(x)$ and $\varphi_{-}(x)$ for the creation and annihilation part of $\varphi(x)$. So that

$$
\begin{aligned}
& \varphi_{+}(x)=(4 \pi)^{-\frac{1}{2}} \int e^{i k x} a^{*}(-k) \mu(k)^{-\frac{1}{2}} d k, \\
& \varphi_{-}(x)=(4 \pi)^{-\frac{1}{2}} \int e^{i k x} a(k) \mu(k)^{-\frac{1}{2}} d k .
\end{aligned}
$$

Scalar boson interactions in two space time dimensions with $V(\alpha)$ a polynomial have recently been extensively studied by Nelson, Glimm, Jaffé, Rosen, and Segal (see Refs. $[1-4,10-13]$ ). For $V(\alpha)=\lambda \alpha^{4}$ Glimm and Jaffé, in a series of papers [2-4], have carried the study very far and showed that one has a quantum field theory satisfying most of the axioms. For $V(\alpha)$ a semi-bounded polynomial Glimm [1] showed that $H$ was bounded below and Rosen [11] proved the essential self-adjointness of $H$, which is essential for getting rid of the space cut-off. We should also mention that Glimm, Jaffé and Rosen uses a smooth space cut-off instead of the sharp cut-off used by this author.

The method used by Glimm, Jaffé and Rosen for proving self-adjointness is to approximate $H$ by the corresponding Hamiltonian in a box $H_{n}$ where only modes of frequency smaller than $n$ is taken to interact. By using function space integration one then obtains uniform estimates for $H_{n}$ which permit one to prove that $H$ is essentially self-adjoint.

Having proved the essential self-adjointness one gets rid of the space cut-off by a theorem of Segal [Ref. 13, Theorem 3].

We shall keep notations close to the notations in the papers by Glimm and Jaffé [2-3] and by Rosen [11], and we shall make use of some of their results, although our method for proving essential self-adjointness is essentially different.

Our reason for considering interactions of the form (1.1) is that we want to give an example of a simple model of a relativistic quantum field theory which have no obvious perturbation theoretical counterpart.

\section{The Interaction $V$}

For any $h$ in $\mathscr{F}^{1}$ we introduce the annihilation-creation operator $a^{\sharp}(h)$, where $a^{\sharp}$ stands for $a^{*}$ or $a$, by

$$
a^{\sharp}(h)=\int a^{\sharp}(k) h(k) d k .
$$

It is well known that $a^{\sharp}(h)$ are closed operators with domain containing the domain of $\left(H_{0}+1\right)^{\frac{1}{2}}$, such that $a^{*}(h)$ and $a(\bar{h})$ are adjoints which have the same domain for $h$ fixed. $a^{\sharp}(h) a^{\sharp}(g)$ have domain containing the 
domain of $H_{0}$, and on the domain of $H_{0}$ we have the commutation relation

$$
\left[a(\bar{h}), a^{*}(g)\right]=(h, g) .
$$

A vector in $\mathscr{F}$ of the form $e^{a^{*}(h)} \Omega_{0}=\sum_{n=0} \frac{1}{n !} a^{*}(h)^{n} \Omega_{0}$, where $\Omega_{0}$ is the Fock vacuum will be called an exponential vector and will be denoted by $e^{h}$. A direct verification shows that $e^{h}$ is in fact a vector in $\mathscr{F}$, and for the inner product of two exponential vectors we have the formula

$$
\left(e^{h}, e^{g}\right)=e^{(h, g)} .
$$

Lemma 2.1. Let $\mathscr{H}_{0}$ be the real vectorspace consisting of all real linear combinations of elements in an orthonormal base in $\mathscr{F}^{1}$. Then the set of all exponential vectors $e^{h}$ with $h$ in $\mathscr{H}_{0}$ spans a dense set in $\mathscr{F}$.

Proof. Let $\left\{h_{i}\right\}$ be the orthonormal base characterizing $\mathscr{H}_{0}$. Consider the exponential vector $e^{g}$ where $g=\sum_{j=1}^{n} s_{j} h_{i_{j}}$, we then see that the strong partial derivative of $e^{g}$ with respect to $s_{1}, \ldots, s_{n}$ at $s_{1}=\cdots=s_{n}=0$ is $a^{*}\left(h_{i_{j}}\right) \ldots a^{*}\left(h_{i_{n}}\right) \Omega_{0}$, which we know spans a dense set in $\mathscr{F}$. On the other hand the strong partial derivative is obtained as a strong limit of linear combinations of exponentials of elements in $\mathscr{H}_{0}$. This proves the lemma.

We shall now give the precise meaning to (1.). Namely

$$
V=\int_{|x| \leqq r} d x \int d v(s) e^{s \varphi+(x)} e^{s \varphi-(x)} .
$$

It is easy to see that (2.2) defines $V$ as a densely defined symmetric form on $\mathscr{F}$. We get namely by using the commutation relations for $a^{\sharp}$ that

$$
\begin{aligned}
& \left(e^{h}, V e^{g}\right)=\int_{|x| \leqq r} \int d v(s) e^{s\left(\bar{h}_{x}+g_{x}\right)} e^{(h, g)} \\
& \left(e^{h}, V e^{g}\right)=\int_{|x| \leqq r} V\left(\bar{h}_{x}+g_{x}\right) d x e^{(h, g)},
\end{aligned}
$$

where we have used the notation $V(\alpha)$ for the analytic continuation of $V(\alpha)$, and $h_{x}=(4 \pi)^{-\frac{1}{2}} \int e^{i k x} h(k) \mu(k)^{-\frac{1}{2}} d k$. From (2.3) we see that if $h_{x}$ and $g_{x}$ are uniformly bounded for $|x| \leqq r$, then $e^{h}$ and $e^{g}$ is in the domain of the bilinear form (2.2). To see that $V$ is a densely defined bilinear form we have only to take an orthonormal basis of functions that goes rapidly to zero and use Lemma 2.1. That $V$ is symmetric follows from (2.3) and the fact that $V(\alpha)$ is real for real $\alpha$.

Let us now assume that the basis which gives $\mathscr{H}_{0}$ in Lemma 2.1 consists of function in $L_{1}(R)$ satisfying $h(k)=\overline{-h(-k)}$, hence for $g \in \mathscr{H}_{0}$, $g_{x}=(4 \pi)^{-\frac{1}{2}} \int e^{i k x} g(k) \mu(k)^{-\frac{1}{2}} d k$ is purely imaginary and uniformly bounded and continuous in $x$. 
Lemma 2.2. $V$ is a positive symmetric operator, which is essentially self-adjoint on the linear span of exponential vectors $e^{h}$ with $h$ in $\mathscr{H}_{0}$. Moreover $V$ commutes with the operator $e^{i \varphi(g)}, \varphi(g)=\int \varphi(x) g(x) d x$.

Proof. Let $h$ be in $\mathscr{H}_{0}$. Let us compute $V e^{h}$ and show that it is in Fock-space

Hence

$$
V e^{h}=\int_{|x| \leqq r} d x \int d v(s) e^{s \varphi_{+}(x)} e^{h} e^{s h_{x}} \Omega_{0} .
$$

$$
\left\|V e^{h}\right\|^{2}=\int_{|y| \leqq r} d y \int d v(s) \int d v(t) e^{t \bar{h}_{y}+s h_{x}} e^{\frac{s t}{2 \pi} K_{0}(m(x-y))} e^{(h, h)}
$$

where

$$
K_{0}(m x)=\frac{1}{2} \int_{-\infty}^{\infty} \frac{e^{i k x}}{\mu(k)} d k
$$

$K_{0}(m)$ is a smooth function away from $x=0$, and

$$
K_{0}(m x)=-\ln \frac{m}{2}|x|+\gamma+o(x) \quad \text { as } \quad|x| \rightarrow 0,
$$

where $\gamma$ is Euler's constant and $o(x)$ represents an absolute convergent power series.

Since $h_{x}$ and $\bar{h}_{y}$ is purely imaginary we get

$$
\begin{aligned}
\left\|V e^{h}\right\|^{2} & \leqq \int d x \int d y \int d v(s) \int d v(t) e^{\frac{s t}{2 \pi} K_{0}((x-y))} e^{(h, h)} \\
& =\int_{|x-y| \geqq \varepsilon}+\int_{|x-y|<\varepsilon} .
\end{aligned}
$$

The first integral is obviously bounded and the second integral is bounded by

$$
2 r \int_{|x|<\varepsilon} d x \int d v(s) \int d v(t) e^{\frac{s t}{2 \pi} K_{0}(m x)}
$$

Choose $\varepsilon$ so small that $K_{0}(m x)>0$ for $|x|>\varepsilon$. Such an $\varepsilon$ exists by (2.4). But then we get that the integral is bounded by

$$
2 r\|v\|^{2} \int_{|x|<\varepsilon} e^{\alpha \mathrm{K}_{0}(m x)} d x
$$

since $v$ has support in an interval of the form $\{s:|s|>\sqrt{2 \pi \alpha}\}$, with $\alpha<1$. This integral is finite by (2.4). This proves that $V$ is an operator which is defined on the linear span of the vectors of the form $e^{h}$. We shall now see that $V$ is a positive operator. By (2.3)

Since

$$
\left(e^{h}, V e^{g}\right)=\int_{|x| \leqq r} V\left(\bar{h}_{x}+g_{x}\right) d x e^{(h, g)} .
$$

$$
V(i \alpha)=\int e^{i \alpha s} d v(s)
$$


is a positive definite function of $\alpha$, and since $h_{x}$ and $g_{x}$ are purely imaginary, we see that for $h_{1}, \ldots, h_{n}$ in $\mathscr{H}_{0}, \int_{|x| \leqq r} V\left(\left(\bar{h}_{i}\right)_{x}+\left(h_{j}\right)_{x}\right) d x$ is a positive definite matrix. That $e^{\left(h_{l}, h_{j}\right)}$ is a positive definite matrix follows from the fact that the inner product in $\mathscr{F}$ is positive definite. Hence $\left(e^{h_{i}}, V e^{h_{j}}\right)$ being the component wise product of two positive definite matrices, is also a positive definite matrix. Hence set $\psi=\Sigma C_{i} e^{h_{i}}$, then

$$
(\psi, V \psi)=\sum_{i j} \bar{C}_{i} C_{j}\left(e^{h_{\imath}}, V e^{h_{j}}\right) \geqq 0 .
$$

This proves that $V$ is a positive operator.

We see that for $g$ real $e^{i \varphi(g)}$ maps exponential vectors into exponential vectors. To see that $V$ commutes with $e^{i \varphi(g)}$ reduces therefore to the evaluation of $\left(e^{i \varphi(g)} e^{h}, V e^{i \varphi(g)} e^{h}\right)$. By (2.2) we get that this is equal to

$$
e^{(h, h)} \int_{|x| \leqq r} V(0) d x=2 r V(0) e^{(h, h)}
$$

which is independent of $g$, and this proves that $V$ commutes with $e^{i \varphi(g)}$.

Let us now introduce the spectral representation of $\mathscr{F}$ with respect to the maximal abelian algebra generated by $e^{i \varphi(g)}$. $\mathscr{F}$ is then represented as an $L_{2}$-space with respect to some measure space. We may choose the measure $d Q$ such that the Fock-vacuum is represented by the function 1. In this $L_{2}(d Q)$ representation the algebra generated by $e^{i \varphi(g)}$ is the algebra of multiplication operators. We remark that the algebra generated by $e^{i \varphi(g)}$ is the same as the algebra generated by $e^{i\left(a^{*}(h)+a(\bar{h})\right)}$ for $h$ in $\mathscr{F}^{1}$, such that $h(p)=\bar{h}(-p)$. Since $\left\|V \Omega_{0}\right\|<\infty$, we see that $V$ is represented by multiplication with the function $V(Q)$ which is in $L_{2}$. Since $e^{h}$ with $h$ in $\mathscr{H}_{0}$, span a dense set in $L_{2}$ and $V$ is a multiplication operator it follows that $V$ is essentially self-adjoint. This proves the lemma.

Lemma 2.3. $H_{0}+V$ is a densely defined positive symmetric operator.

Proof. We have seen that $V$ is positive and symmetric. So the only thing we have to prove is that $H_{0}$ and $V$ has a common dense domain. We have seen that $e^{h}$ for $h$ in $\mathscr{H}_{0}$ is in the domain of $V$. It is easy to see that if $h(p)$ has compact support then $e^{h}$ is also in the domain of $H_{0}$. By Lemma 2.1 however $e^{h}$ for $h$ with compact support and $h$ in $\mathscr{H}_{0}$ spans a dense set in $\mathscr{F}$. This proves the lemma.

\section{The Hamiltonian $\mathrm{H}=\mathrm{H}_{\mathbf{0}}+\mathrm{V}$}

In this section we shall prove that $H=H_{0}+V$ is essentially selfadjoint, on a domain contained in the intersection of the domains of $H_{0}$ and of $V$. In order to prove this we shall need the $L_{2}(d Q)$ representation of $\mathscr{F}$ introduced in the last paragraph. 
A bounded operator $A$ on an $L_{2}$-space is said to have a positive, ergodic kernel if whenever $\psi$ and $\chi$ are non negative $L_{2}$-functions with non zero norm, we have that $0 \leqq(\psi, A \chi)$ and for some $j$ depending on $\psi$ and $\chi, 0<\left(\psi, A^{j} \chi\right)$.

We shall need the following lemma which is proved by Glimm and Jaffé [Ref. 3, Lemma 2.3.2].

Lemma 3.1. $e^{-t H_{0}}$ has a positive, ergodic kernel on $L_{2}(d Q)$ for all values of $t>0$.

Lemma 3.2. $e^{-t H_{0}}$ is a contraction semigroup on $L_{p}(d Q)$ for $1 \leqq p \leqq \infty$.

Proof. Since $H_{0}$ is a positive operator on $L_{2}, e^{-t H_{0}}$ is a contraction semi group on $L_{2}$. Let $\psi \in L_{2}$ then $\left\|e^{-t H_{0}} \psi\right\|_{1}=\int\left|\left(e^{-t H_{0}} \psi\right)(Q)\right| d Q$ $\leqq \int\left(e^{-t H_{0}}|\psi|\right)(Q) d Q$, by the triangle inequality and the fact that $e^{-t H_{0}}$ has a positive kernel. On the other hand we have

$$
\int\left(e^{-t H_{0}}|\psi|\right)(Q) d Q=\left(\Omega_{0}, e^{-t H_{0}}|\psi|\right)=\left(\Omega_{0},|\psi|\right)=\|\psi\|_{1} .
$$

Since $L_{2}$ is dense in $L_{1}$ we see that $e^{-t H_{0}}$ is a contraction semigroup on $L_{1}$. Since $e^{-t H_{0}}$ is symmetric on $L_{2}$ with a positive kernel, we get by duality that $e^{-t H_{0}}$ is a contraction semigroup on $L_{\infty}$. The Riesz Thorin convexity theorem then gives us that $e^{-t H_{0}}$ is a contraction semigroup on $L_{p}, 1 \leqq p \leqq \infty$. This proves the lemma.

In the previous paragraph we proved that $V$ was a positive selfadjoint operator which commute with operators of the form $e^{i \varphi(h)}$ and that $\Omega_{0}$ is in the domain of $V$. Since the $L_{2}(d Q)$ representation is a spectral representation of the maximal abelian algebra generated by $e^{i \varphi(h)}$, we see that in this representation $V$ is represented by a multiplication operator $V(Q)$, where $V(Q)$ is a positive function in $L_{2}(d Q),\|V\|_{2}=\|V \Omega\|$.

Let $V_{k}(Q)$ if $V(Q)<k$ and $V_{k}(Q)=k$ if not. Let $V_{k}$ be the multiplication operator given by $V_{k}(Q)$, and set $H_{k}=H_{0}+V_{k}$. Since $V_{k}$ is positive, symmetric and bounded we see that $H_{k}$ is positive and self-adjoint with the same domain as $H_{0}$.

Theorem 3.1. Let $\tilde{H}$ be the Friedrichs extension of $H$. Then $e^{-t H_{k}}$ converge strongly to $e^{-t \tilde{H}}$ as $k$ tends to infinity, and the convergence is uniform in $t$ for $t$ on compact subsets.

Proof. We have obviously that

$$
0 \leqq H_{k} \leqq H_{k^{\prime}} \leqq \tilde{H}
$$

for $k \leqq k^{\prime}$. Moreover for $\psi$ and $\chi$ in $D\left(\tilde{H}^{\frac{1}{2}}\right)$ we have that $\left(\psi, H_{k} \chi\right) \rightarrow(\psi, \tilde{H} \chi)$. Using then the theorem on convergence from below of symmetric semibounded forms (Theorem 3.13, Ch. VIII, Ref. [9]) we get that $\left(z-H_{k}\right)^{-1}$ converge strongly to $(z-\tilde{H})^{-1}$ for $\operatorname{Re} z<0$. The semigroup convergence 
theorem (Theorem 2.16, Ch. IX, Ref. [9]) then gives us that $e^{-t H_{k}}$ converge strongly to $e^{-t \tilde{H}}$ uniformly in $t$ on compacts. This proves the theorem.

Lemma 3.3. $e^{-t \tilde{H}}$ has a positive kernel on $L_{2}(d Q)$, and $e^{-t \tilde{H}}$ is a contraction semigroup on $L_{p}(d Q)$, for $1 \leqq p \leqq \infty$.

Proof. That $e^{-t \tilde{H}}$ has a positive kernel means that for $\psi$ and $\chi$ positive and in $L_{2},\left(\psi, e^{-t \tilde{\boldsymbol{H}}} \chi\right) \geqq 0$. By Theorem 3.1 it is enough to prove that $\left(\psi, e^{-t H_{k}} \chi\right) \geqq 0$ for all $k$. Since $V_{k}$ is bounded we get by the Trotter-Kato product formula

$$
e^{-t H_{k}}=\lim _{n \rightarrow \infty} e^{\frac{-t}{n} H_{0}} e^{\frac{-t}{n} V_{k}} \ldots e^{\frac{-t}{n} H_{0}} e^{\frac{-t}{n} V_{k}},
$$

that $e^{-t H_{k}}$ has a positive kernel. Because $e^{\frac{-t}{n} V_{k}}$ maps positive functions into positive function, and by Lemma $3.1 e^{\frac{-t}{n} H_{0}}$ also maps positive functions into positive functions. Hence $e^{-t^{t H_{k}}} \chi$ is the strong limit of positive functions, and therefore $e^{-t H_{k}} \chi \geqq 0$. This proves the first part of the lemma.

To prove the second part we see from (3.1) that $e^{-t H_{k}}$ is a contraction on $L_{\infty}(d Q)$, since both $e^{\frac{-t}{n} H_{0}}$ and $e^{\frac{-t}{n} V_{k}}$ are contractions on $L_{\infty}(d Q)$. Let $\psi$ be in $L_{\infty}(d Q)$. By Theorem 3.1, $\chi=e^{-t H} \psi$ is then the $L_{2}$-limit of $\chi_{k}=e^{-t H_{k}} \chi$. Therefore there exist a subsequence $\chi_{n}$ such that $\chi_{n}$ converge to $\chi$ almost everywhere. Since $e^{-t H_{k}}$ is a contraction on $L_{\infty}$ we have that $\left\|\chi_{k}\right\|_{\infty} \leqq\|\psi\|_{\infty}$, so that $\left|\chi_{n}(Q)\right| \leqq\|\psi\|_{\infty}$, almost everywhere, therefore $\|\chi\|_{\infty} \leqq\|\psi\|_{\infty}$. This proves the lemma.

Theorem 3.2. $H=H_{0}+V$ is essentially self-adjoint on the domain $(z-\tilde{H})^{-1} L_{\infty} \subset D\left(H_{0}\right) \cap D(V)$, hence $\tilde{H}$ is the closure of $H$.

Proof. Since $e^{-t \tilde{H}}$ is a contraction semigroup on $L_{\infty}$ we see that $(z-\tilde{H})^{-1} L_{\infty} \subseteq L_{\infty}$ for $\operatorname{Re} z<0$.

Since $L_{\infty}$ is dense in $L_{z}$ we see that $(z-\tilde{H})^{-1} L_{\infty}$ is a core for $\tilde{H}$. Since $V$ is in $L_{2}$ we have $(z-\tilde{H})^{-1} L_{\infty} \leqq L_{\infty} \subseteq D(V)$. To prove the theorem it is therefore enough to prove that $(z-\tilde{H})^{-1} L_{\infty}$ is contained in $D\left(H_{0}\right)$, because as we have seen $\tilde{H}$ is essentially self-adjoint on $(z-\tilde{H})^{-1} L_{\infty}$. Since $V_{k}$ is a bounded function $\left(z-H_{k}\right)^{-1} L_{\infty} \subseteq D\left(H_{0}\right)$. Let $\varphi \in L_{\infty}$ and set $\psi_{k}=\left(z-H_{k}\right)^{-1} \varphi$. By Theorem $3.1 \psi_{k}$ converge in $L_{2}$ to $\psi=(z-\tilde{H})^{-1} \varphi$. To prove that $\psi$ is in $D\left(H_{0}\right)$, using that $H_{0}$ is closed it is enough to prove that $H_{0} \psi_{k}$ converge in $L_{2}$. We have seen that $e^{-t H_{k}}$ is a contraction semigroup on $L_{\infty}$. This gives us that the norm of $\left(z-H_{k}\right)^{-1}: L_{\infty} \rightarrow L_{\infty}$ is uniformly bounded in $k$, so that $\left\|\psi_{k}\right\|<C$ independent of $k$.

$$
\varphi=\left(z-H_{k}\right) \psi_{k}=\left(z-H_{0}\right) \psi_{k}-V_{k} \psi_{k},
$$


so that it is enough to prove that $V_{k} \psi_{k}$ converge in $L_{2}$.

$$
V_{k} \psi_{k}-V \psi=\left(V_{k}-V\right) \psi+V\left(\psi_{k}-\psi\right) \text {. }
$$

Since $\psi \in L_{\infty},\left\|\left(V_{k}-V\right) \psi\right\|_{2} \leqq\left\|V_{k}-V\right\|_{2}\|\psi\|_{\infty}$ which tends to zero since $V$ is in $L_{2}$.

$$
\begin{aligned}
\left\|V\left(\psi_{k}-\psi\right)\right\|_{2} & \leqq\left\|\left(V-V_{l}\right)\left(\psi_{k}-\psi\right)\right\|_{2}+\left\|V_{l}\left(\psi_{k}-\psi\right)\right\|_{2} \\
& \leqq 2 C\left\|V-V_{l}\right\|_{2}+l\left\|\psi_{k}-\psi\right\|_{2} .
\end{aligned}
$$

By choosing $l$ so large that $\left\|V-V_{l}\right\|_{2} \leqq(4 C)^{-1} \varepsilon$ and then choosing $k$ so large that $\left\|\psi_{k}-\psi\right\|_{2} \leqq(2 l)^{-1} \varepsilon$ we see that $\left\|V\left(\psi_{k}-\psi\right)\right\| \leqq \varepsilon$. This proves that $H_{0} \psi_{k}$ converge strongly to $H_{0} \psi$, and therefore that $(z-\tilde{H})^{-1} L_{\infty}$ is in $D\left(H_{0}\right)$. This proves the theorem.

Having proved that $\tilde{H}$ is the closure of $H=H_{0}+V$, we shall from now on simply write $H$ for $\tilde{H}$.

\section{Existence and Uniqueness of the Vacuum of $H$}

By a vacuum $\Omega$ of $H$ we shall understand an eigenvector $\Omega$ whoose eigenvalue is a lower bound for the spectrum of $H$, and in this section we shall prove that $H$ has a unique vacuum $\Omega$. The technique of Glimm and Jaffé [3] for proving the existence of $\Omega$ will not work due to the fact that $V$ is only in $L_{2}(d Q)$. Using the positivity of $V$ however one may prove a norm approximation lemma (Lemma 4.2), which enables one to extend the technique of Glimm and Jaffé to positive $V$ in $L_{1}(d Q)$.

Lemma 4.1. Let $1<p$ and $r<\infty$. Then there exists a $T$ such that for $T \leqq t e^{-t H_{0}}$ is a contraction from $L_{p}(d Q)$ to $L_{r}(d Q)$. If $p$ is bounded away from 1 and $r$ is bounded then $T$ does not depend on $p$ or $r$.

Proof. For the case of finitely many degrees of freedom this lemma was proved by Glimm (Ref. [1], Lemma 5.1). The extension to infinitely many degrees of freedom is strait forward and is also given in Ref. [12], Theorem III.18.

Lemma 4.2. Let $V_{1}$ and $V_{2}$ be two nonnegative functions in $L_{2}(d Q)$, and let $H_{1}$ and $H_{2}$ be the unique self-adjoint extentions of $H_{0}+V_{1}$ and $H_{0}+V_{2}$. Then there is a positive real $T$ independent of $V_{1}$ and $V_{2}$ such that for $t \geqq T$

$$
\left\|e^{-t H_{1}}-e^{-t H_{2}}\right\| \leqq\left[1-e^{-t\left\|V_{1}-V_{2}\right\|_{1}}\right]^{\frac{1}{4}} .
$$

Proof. Let $X$ be the compact space that carries the measure $d Q$, and let $Y_{n}$ be the product of $X$ with itself $n+1$-times. On $Y_{n}$ we introduce the measure $d \mu_{n}$, where $d \mu_{n}$ is characterized by the relation

$$
\int F_{0}\left(Q_{0}\right) \cdots F_{n}\left(Q_{n}\right) d \mu_{n}=\left(\Omega_{0}, e^{-\frac{t}{n} H_{0}} F_{0} \cdots e^{-\frac{t}{n} H_{0}} F_{n} \Omega_{0}\right)
$$

18 Commun. math. Phys., Vol. 21 
for $F_{0} \cdots F_{n}$ in $L_{2}(d Q)$. It follows from the fact that $e^{-\frac{t}{n} H_{0}}$ has a positive kernel, that the relation above defines a unique measure $d \mu_{n}$ on $Y_{n}$.

Let now $\varphi$ and $\psi$ be in $L_{2}(d Q)$, then by the Trotter-Kato product

formula (3.1)
$\left(\varphi_{1}\left(e^{-t H_{1}}-e^{-t H_{2}}\right) \psi\right)=\lim _{n \rightarrow \infty} \int d \mu_{n} \bar{\varphi}\left(Q_{0}\right) \psi\left(Q_{n}\right)\left[e^{-\frac{t}{n} \sum_{i=1}^{n} V_{1}\left(Q_{i}\right)}-e^{-\frac{t}{n} \sum_{i=1}^{n} V_{2}\left(Q_{i}\right)}\right]$

By Hølders inequality we get

$$
\begin{aligned}
\int d \mu \bar{\varphi}\left(Q_{0}\right) \psi\left(Q_{n}\right)\left[e^{-\frac{t}{n} \sum_{i=1}^{n} V_{1}\left(Q_{i}\right)}-e^{-\frac{t}{n} \sum_{i=1}^{n} V_{2}\left(Q_{i}\right)}\right] \\
\leqq\left\|\bar{\varphi}\left(Q_{0}\right) \psi\left(Q_{n}\right)\right\|_{4 / 3} \cdot\left\|e^{-\frac{t}{n} \sum_{i=1}^{n} V_{1}\left(Q_{i}\right)}-e^{-\frac{t}{n} \sum_{i=1}^{n} V_{2}\left(Q_{i}\right)}\right\|_{4} .
\end{aligned}
$$

By the definition of $d \mu_{n}$

$$
\begin{aligned}
\left\|\bar{\varphi}\left(Q_{0}\right) \psi\left(Q_{n}\right)\right\|_{p}^{p} & =\left(\Omega_{0},|\varphi|^{p} e^{-t H_{0}}|\psi|^{p} \Omega_{0}\right) \\
& =\left(e^{-\frac{t}{2} H_{0}}|\varphi|^{p} \Omega_{0}, e^{-\frac{t}{2} H_{0}}|\psi|^{p} \Omega_{0}\right) \\
& \leqq\left\|e^{-\frac{t}{2} H_{0}}|\varphi|^{p} \Omega_{0}\right\|_{2} \cdot\left\|e^{-\frac{t}{2} H_{0}}|\psi|^{p} \Omega_{0}\right\|_{2} .
\end{aligned}
$$

From Lemma 4.1 we get that for any $q>1$ there is a $T$ such that for $t \geqq T$

$$
\left\|e^{-\frac{t}{2} H_{0}}|\varphi|^{p} \Omega_{0}\right\|_{2} \leqq\left\||\varphi|^{p}\right\|_{q}=\|\varphi\|_{p q}^{p},
$$

and with $p=4 / 3$ and $q=6 / 4$ we see that

$$
\left\|\bar{\varphi}\left(Q_{0}\right) \psi\left(Q_{n}\right)\right\|^{4 / 3} \leqq\|\varphi\|_{2}\|\psi\|_{2}
$$

Using that $V_{1}$ and $V_{2}$ are positive we get

$$
\begin{aligned}
\left\|e^{-\frac{t}{n} \sum_{i=1}^{n} V_{1}\left(Q_{i}\right)}-e^{-\frac{t}{n} \sum_{i=1}^{n} V_{2}\left(Q_{i}\right)}\right\|_{4}^{4} & \leqq e^{-\frac{t}{n} \sum_{i=1}^{n} V_{1}\left(Q_{i}\right)}-e^{-\frac{t}{n} \sum_{i=1}^{n} V_{2}\left(Q_{2}\right)} \|_{1} \\
& \leqq 1-e^{-\frac{t}{n} \sum_{i=1}^{n}\left|V_{1}\left(Q_{i}\right)-V_{2}\left(Q_{i}\right)\right|} \|_{1} \\
& =1-\int d \mu_{n} e^{-\frac{t}{n} \sum_{i=1}^{n}\left|V_{1}\left(Q_{i}\right)-V_{2}\left(Q_{i}\right)\right|}
\end{aligned}
$$

By Jensens inequality this is bounded by

$$
1-e^{-\frac{t}{n} \int d \mu_{n} \sum_{i=1}^{n}\left|V_{1}\left(Q_{i}\right)-V_{2}\left(Q_{i}\right)\right|}=1-e^{-t\left\|V_{1}-V_{2}\right\|_{1}} .
$$

This together with (4.4), (4.3) and (4.2) proves the lemma. 
Theorem 4.1. $H$ has a unique vacuum $\Omega$, and let $\omega$ be its eigenvalue. Then for any $\varepsilon>0 H$ has a finite spectrum in $[\omega, \omega+m-\varepsilon]$.

Proof. To prove that $H$ has finite spectrum in $[\omega, \omega+m-\varepsilon]$ it is enough to prove that $e^{-t H}$ has a finite spectrum in the interval $\left[e^{-t(\omega+m-\varepsilon)}, e^{-t \omega}\right]$, for a $t \geqq T$. By Lemma $4.2 e^{-t H_{k}}$ converge in norm to $e^{-t H}$ for $t \geqq T$. Using now that norm limits of compact operators are compact, it is enough to prove that the restriction of $e^{-t H_{k}}$ to $\left[e^{-t(\omega+m-\varepsilon)}, e^{-t \omega}\right]$ is a compact operator, or that $H_{k}$ has a finite spectrum in $[\omega, \omega+m-\varepsilon]$ for $k$ large. Since however $H_{k}=H_{0}+\dot{V}_{k}$ where $V_{k}$ is a bounded function, one may here use the method of Glimm and Jaffe [3] to prove that $H_{k}$ has a finite spectrum in $\left[\omega_{k}, \omega_{k}+m-\varepsilon\right]$ where $\omega_{k}$ is the lower bound on $H_{k}$. Due to the normconvergence of $e^{-t H_{k}}$ we have that $\omega_{k}$ converge to $\omega$, and this then gives us that $H$ has a finite spectrum in $[\omega, \omega+m-\varepsilon]$. This gives also the existence of $\Omega$. That $\Omega$ is unique is proved by showing $e^{-t H}$ has a positive ergodic kernel. The proof that $e^{-t H}$ has a positive ergodic kernel is just a trivial modification of the proof given by Glimm and Jaffé that in the case of the space cut-off $: \varphi^{4}$ : interaction $e^{-t H}$ has a positive ergodic kernel. We shall therefore not produce the proof but rather refer the reader to Glimm and Jaffé (Ref. [3], § 2.3).

\section{Removal of the Space Cut-Off}

In a series of papers [2-4] Glimm and Jaffé have shown for the $: \varphi^{4}$ : model in two dimensions that one may also remove the space cut-off. And they show that the theory with all cut-off's removed satisfy most of the axioms of the Haag-Kastler scheme [5], and several of the Wightman axioms [14]. But their methods are mostly not restricted to $: \varphi^{4}:$. As a consequence most of their work carries over to the general class of models we are stydying.

First we obtain a dynamics, independent of the cut-off, for bounded functions of the free fields localized in a bounded region of space. More precisely, lęt $R_{a, b}$ be the von Neumann algebra of operators generated by the spectral projections of all the fields $\varphi(f)$ and $\pi(f)$ with supp $f \subset[a, b]$, where $\pi$ is the canonical conjugate field to $\varphi$,

$$
\pi(x)=i(4 \pi)^{-\frac{1}{2}} \int e^{i k x}\left[a^{*}(k)-a(-k)\right] \mu(k)^{\frac{1}{2}} d k .
$$

The time translate of $A$ in $R_{a, b}$ is then given by

$$
\alpha_{t}(A)=e^{i t H_{r}} A e^{-i t H_{r}},
$$


with $H_{r}$ the closure of $H_{0}+V_{r}, V_{r}=\int_{|x| \leqq r}: V(\varphi(x)): d x$. Due to the essential self-adjointness of $H_{r}$, we then get by a theorem of Segal [13, Theorem 3] that $\alpha_{t}(A)$ is independent for $r$ for $r \geqq \max \{|a|,|b|\}+|t|$. This then removes the spatial cut-off as far as the dynamics of local algebras is concerned, and the resulting theory is local.

Following Glimm and Jaffé [3] the space cut-off may be completely removed from the theory with the aid of the vacuum vectors $\Omega_{r}$. For this however we need the following lemma.

Lemma 5.1.

$$
\left(\Omega_{r}, H_{0} \Omega_{r}\right) \leqq 2 r\|v\|
$$

where $v$ is the measure describing the interaction.

Proof. Since $V_{r}$ is positive we have

$$
\begin{aligned}
\left(\Omega_{r}, H_{0} \Omega_{r}\right) & \leqq\left(\Omega_{r}, H_{r} \Omega_{r}\right) \leqq\left(\Omega_{0}, H_{r} \Omega_{0}\right) \\
& =\left(\Omega_{0}, V_{r} \Omega_{0}\right)=2 r\|v\|,
\end{aligned}
$$

where we use that $\Omega_{r}$ is the vacuum for $H_{r}$. This proves the lemma.

From this lemma we get that the free energy densities of the vacuums $\Omega_{r}$ are uniformly bounded, and therefore also that the particle number densities also are uniformly bounded. As shown by Glimm and Jaffé, this is enough, after a slight modification of $\Omega_{r}$ by averaging over space, to ensure that a subsequence of the vacuums converge weakly as states on the local $C^{*}$-algebra $\mathscr{A}$ of bounded functions of the local fields. In this way one obtain a state $\varrho$ on $\mathscr{A}$ which is invariant under translation as well in space as in time. The physical Hilbert space $\mathscr{F}_{\text {ren }}$ is then the representation space for the representation of $\mathscr{A}$ induced by $\varrho$. Since $\varrho$ is invariant under $\alpha_{t}$ the equation

$$
U^{*}(t) A_{\mathrm{ren}} U(t)=\left(\alpha_{t}(A)\right)_{\mathrm{ren}}
$$

defines a unitary group $U(t) . A_{\text {ren }}$ is the representation of $A$ in $\mathscr{F}_{\text {ren }}$. It follows also that the representation given by $\varrho$ is locally Fock and that the infinitesimal generator $H_{\text {ren }}$ of $U(t)$ is positive. That $\mathscr{F}_{\text {ren }}$ is locally Fock enables one to define the physical fields and to establish local properties of the physical theory by working in Fock space. For more details about the consequences of Lemma 5.1 we refer the reader to Glimm and Jaffé $[3,4]$.

It is a pleasure for me to thank B. Simon for many discussions and valuable comments on the subject of this paper. I am also grateful to A. S. Wightman for arranging my stay at Joseph Henry Laboratories, Princeton University, and for his helpfulness during my stay there, during which this paper was written. 


\title{
References
}

1. Glimm, J.: Boson fields with nonlinear self-interaction in two dimensions. Commun. math. Phys. 8, 12-25 (1968).

2. - Jaffé, A.: A $\lambda \varphi^{4}$ quantum field theory without cutoffs, I. Phys. Rev. 176, 1945-1951.

3. - - A $\lambda\left(\varphi^{4}\right)_{2}$ quantum field theory without cutoffs, II. The field operators and the approximate vacuum. Ann. Math. 91, 362 (1970).

4. - - A $\lambda \varphi^{4}$ quantum field theory without cutoffs, III, to appear.

5. Haag, R., Kastler, D.: An algebraic approach to quantum field theory. J. Math. Phys. 5, 848-861 (1964).

6. - Schroer, B.: Postulates of quantum field theory. J. Math. Phys. 3, 248-256 (1962).

7. Høegh-Krohn, R.: On the spectrum of the space cut-off $: \varphi^{4}:$ Hamiltonian in two spacetime dimensions. Preprint, Princeton University.

8. - On the spectrum of the space cut-off :P( $\varphi)$ : Hamiltonian in two space-time dimensions. Preprint, Princeton University.

9. Kato,T.: Perturbation theory for linear operations. Berlin-Heidelberg-New York: Springer 1965.

10. Nelson, R.: A quartic interaction in two dimensions. Mathematical theory of elementary particles, ed. by R.Goodman and I.Segal, pp. 69-73. Cambridge: M.I.T. Press 1965.

11. Rosen, L.: A $\lambda \varphi^{2 n}$ field theory without cutoffs. Preprint, New York University.

12. Simon, B., Høegh-Krohn, R.: Hypercontractive semigroups and two dimensional selfcoupled bose fields. Preprint, Princeton University.

13. Segal, I.: Notes toward the construction of nonlinear relativistic quantum fields, I. The Hamiltonian in two space-time dimensions as a generator of $C^{*}$-automorphism group. Proc. Natl. Acad. Sci. U.S. 57, 1178-1183 (1967).

14. Streater,R., Wightman,A.: PCT, spin and statistics, and all that. New York: W.A. Benjamin 1964.

\author{
R. Høegh-Krohn \\ Universitetet I Oslo \\ Matematisk Institutt - Postboks 1053 \\ Blindern - Oslo 3 \\ Norway
}

